\title{
A Comparative Bio-Evaluation and Chemical Profiles of Calendula officinalis L. Extracts Prepared via Different Extraction Techniques
}

\author{
Gunes Ak ${ }^{1}$, Gokhan Zengin ${ }^{1}$ (D), Kouadio Ibrahime Sinan ${ }^{1}$, Mohamad Fawzi Mahomoodally ${ }^{2,3, *}$, \\ Marie Carene Nancy Picot-Allain ${ }^{3}{ }^{\circ}$, Oguz Cakır $^{4}{ }^{\oplus}$, Souheir Bensari ${ }^{5}$, \\ Mustafa Abdullah Yilmaz ${ }^{6}$ (D), Monica Gallo ${ }^{7, *(D)}$ and Domenico Montesano ${ }^{8}$ (D) \\ 1 Department of Biology, Science Faculty, Selcuk University, 42130 Konya, Turkey; \\ akguneselcuk@gmail.com (G.A.); gokhanzengin@selcuk.edu.tr (G.Z.); sinankouadio@gmail.com (K.I.S.) \\ Institute of Research and Development, Duy Tan University, Da Nang 550000, Vietnam \\ 3 Department of Health Sciences, Faculty of Science, University of Mauritius, 230 Réduit, Mauritius; \\ picotcarene@yahoo.com \\ 4 Science and Technology Research and Application Center, Dicle University, 21280 Diyarbakir, Turkey; \\ ocakir44@gmail.com \\ 5 Laboratoire de Génétique, Biochimie et Biotechnologies Végétales GBBV, Faculté des Sciences de la Nature et \\ de la Vie, Université Frères Mentouri Constantine1, Route d'Aïn El Bey, 25017 Constantine, Algeria; \\ souheir.ben@gmail.com \\ 6 Department of Pharmaceutical Chemistry, Faculty of Pharmacy, Dicle University, 21280 Diyarbakir, Turkey; \\ mustafaabdullahyilmaz@gmail.com \\ 7 Department of Molecular Medicine and Medical Biotechnology, University of Naples Federico II, \\ via Pansini, 5, 80131 Naples, Italy \\ 8 Department of Pharmaceutical Sciences, Section of Food Science and Nutrition, University of Perugia, \\ via San Costanzo 1, 06126 Perugia, Italy; domenico.montesano@unipg.it \\ * Correspondence: mohamadfawzimahomoodally@duytan.edu.vn (M.F.M.); mongallo@unina.it (M.G.)
}

Received: 19 July 2020; Accepted: 24 August 2020; Published: 26 August 2020

check for updates

Featured Application: This study provides valuable data on the influence of extraction techniques on the recovery of bioactive compounds from Calendula officinalis useful for the formulation of therapeutic preparations.

\begin{abstract}
Calendula officinalis L., (marigold), well known for its medicinal properties, has been extensively studied for its therapeutic properties. Nonetheless, as far as the literature could establish, no study has attempted to comparatively assess the biological (antioxidant and enzyme inhibitory potential) of the flowers, leaves, and roots of $C$. officinalis extracted using conventional (maceration and Soxhlet extraction (SE)) and non-conventional extraction (homogenizer (HAE) and ultrasound (UAE) assisted extraction) techniques. The detailed phytochemical profile of each extract along with the concentration of specific bioactive compounds has also been established. Total phenolic content was highest for the flower extracts while flavonoid content was highest in the leaf extracts. Phytochemical profiling showed that the extraction method influenced the phytochemical composition of the extract. Nicotiflorin was identified in the flower extracts only while amentoflavone occurred only in the roots, inferring that the occurrence of bioactive compounds varies within a plant. The flower extracts showed highest antioxidant potential while the roots extracts were potent inhibitors of cholinesterase and tyrosinase. This study provides valuable data on the influence of extraction techniques on the recovery of bioactive compounds from plants. In an endeavor to scale-up extraction from plant considering the more efficient extraction method is of paramount importance. Moreover, the study highlighted the necessity to thoroughly examine the biological activities of various parts of a plant obtained via different extraction protocols.
\end{abstract}


Keywords: marigold; maceration; cholinesterase; tyrosinase

\section{Introduction}

Plant-derived bioactive compounds have always served a sustainable resource for drug, nutraceutical and cosmeceutical development. However, the first step in this endeavor, is the selection of the best plant part and an appropriate extraction protocol which yields best activity. In this respect, several conventional and non-conventional extraction methods have been designed and used in a plethora of studies. Among these methods, soxhlet, maceration or percolorization are considered as conventional methods. However, the conventional methods have some disadvantages such as the use of high volume of solvent and long extraction times. In this sense, recently some new and improved techniques have emerged as green or non-conventional extraction techniques. Ultrasound-assisted, homogenizer-assisted, and microwave-assisted extractions are classified as the green extraction methods. The green extraction methods allows working with low volume of solvents as well as shorter extraction times. In addition, these methods gave higher extraction yields. Altogether, the selection of the best extraction method is one of the most popular subjects in the chemical and biological investigations [1,2].

In the present study, Calendula officinalis (Asteraceae), an important species of the genus Calendula and is well known for its medicinal properties [3], was investigated for biological activity based on different extraction protocols. Also known as pot marigold, C. officinalis has been cultivated as a food and medicinal plant since the Middle Ages and the colorant made from this plant was used in the ancient Greece and Rome and by early Indian and Arabic population [4]. In previous studies, the flowers and leaves of $C$. officinalis have been reported as a source of biologically active compounds. For example, in a study conducted by Cruceiru et al [5] described that the presence of hydroxycinnamic acids and flavonols in the flowers and leaves of $C$. officinalis. Similarly, quercertin and apigenin derivatives were found to be in the hydroalcoholic extract of flower [6]. In addition to phenolics, the leaves and flowers contained saponins, triterpenoids, essential oils, and carotenoids [7-11]. C. officinalis seeds are particularly rich in calendic acid which is used in cosmetic formulations. The flowers are exploited as a commercial source of lutein. The cell rejuvenates, healing, anti-inflammatory, soothing and skin softening properties of $C$. officinalis flower support its use in skin care products [3]. The antimicrobial and antifungal properties of $C$. officinalis flower extract support its application for the treatment of inflammation and skin wounds [12]. The application of $C$. officinalis cream balm to diabetic patients' feet prevented infection, reduced itching, redness, dryness, pain, and promoted the disappearance of scars [13]. Favorable proliferation and adhesion to fibroblast cell as well as antibacterial properties was observed while incorporating $C$. officinalis extract to an electrospun fiber scaffold consisting of poly ( $\varepsilon$-caprolactone), zein, and gum Arabic as compared to plain PCL/zein/GA scaffold, thereby suggesting the possible application of $C$. officinalis embedded scaffolds biomaterial for skin regeneration [14]. The Food and Drug Administration (FDA) has classified C. officinalis as a GRAS (Generally Recognized as Safe) substance [15]. Edible Calendula flowers possessing nutritional properties and being rich in quinic acid and $\alpha$-tocopherol, were used for the preparation of omelets, salads, and as accompaniment with cheese in Medieval France [16]. The flower extract was used to manage measles, smallpox; the flower juice was used to treat skin diseases, ulcers, costiveness, jaundice, fever, wounds, and to suppress menstruation, promote sweating, and prevent suppuration; infusion of the plant was used to manage anemia and the ointment was used against bruises, sores, cracks, and cuts in the hands [7]. C. officinalis teas were taken against colitis, gastroduodenitis, and duodenal ulcers [17]. Literature has shown that $C$. officinalis has been extensively studied for its various health properties. Although, considerable research work has been conducted on this plant, no scientific report has comparatively assessed the biological potential of different plant parts of $C$. officinalis. Therefore, the present study aims at investigating the biological properties of methanol extracts of the flowers, leaves, and roots of 
C. officinalis. Maceration and Soxhlet extraction, categorized as conventional extraction methods, and homogenizer and ultrasound assisted extractions, categorized as non-conventional extraction methods, were used.

\section{Materials and Methods}

\subsection{Plant Material and Extracts Preparation}

The raw plant material was obtained in a field study (in a wild population) of Bosna-Hersek location (Selcuklu, Konya, Turkey), in April 2020. The plant name was confirmed by one botanist, Dr. Evren Yildiztugay and one species were kept as voucher specimen in the herbarium (KNYA). The flowers, leaves and roots carefully separated and then the plant samples were kept in 10 day in a shade for 10 days to dry. A laboratory mill was used to powder these plant samples (particle size about $2 \mathrm{~mm}$ ).

To obtain extracts, homogenizer-assisted (HAE), maceration (MAC), soxhlet (SOX), and ultrasound-assisted (UAE) extraction methods were used. Methanol (purchased from Merck, Darmstadt, Germany), was used as a solvent in these methods. HAE was performed using ultra-turrax (IKA- T25, IKA®-Werke GmbH \& Co. KG, Staufen, Germany), while UAE was performed in an ultrasound bath (Daihan, WUC-D10H, Wonju-si, Korea). MAC and SOX were selected as conventional methods and they were performed by using one magnetic stir (Daihan, Plate-type MSH-20D, Wonju-si, Korea) and soxhlet apparatus (Isolab, Istanbul, Turkey), respectively. We used the ratio $1 / 20$ (plant/solvent) in these methods. The details of extraction procedures are summarized in Figure 1. The extracts were filtered and using one rotary-evaporator was used to remove solvents. Obtained extracts were kept at $4{ }^{\circ} \mathrm{C}$ until analysis.

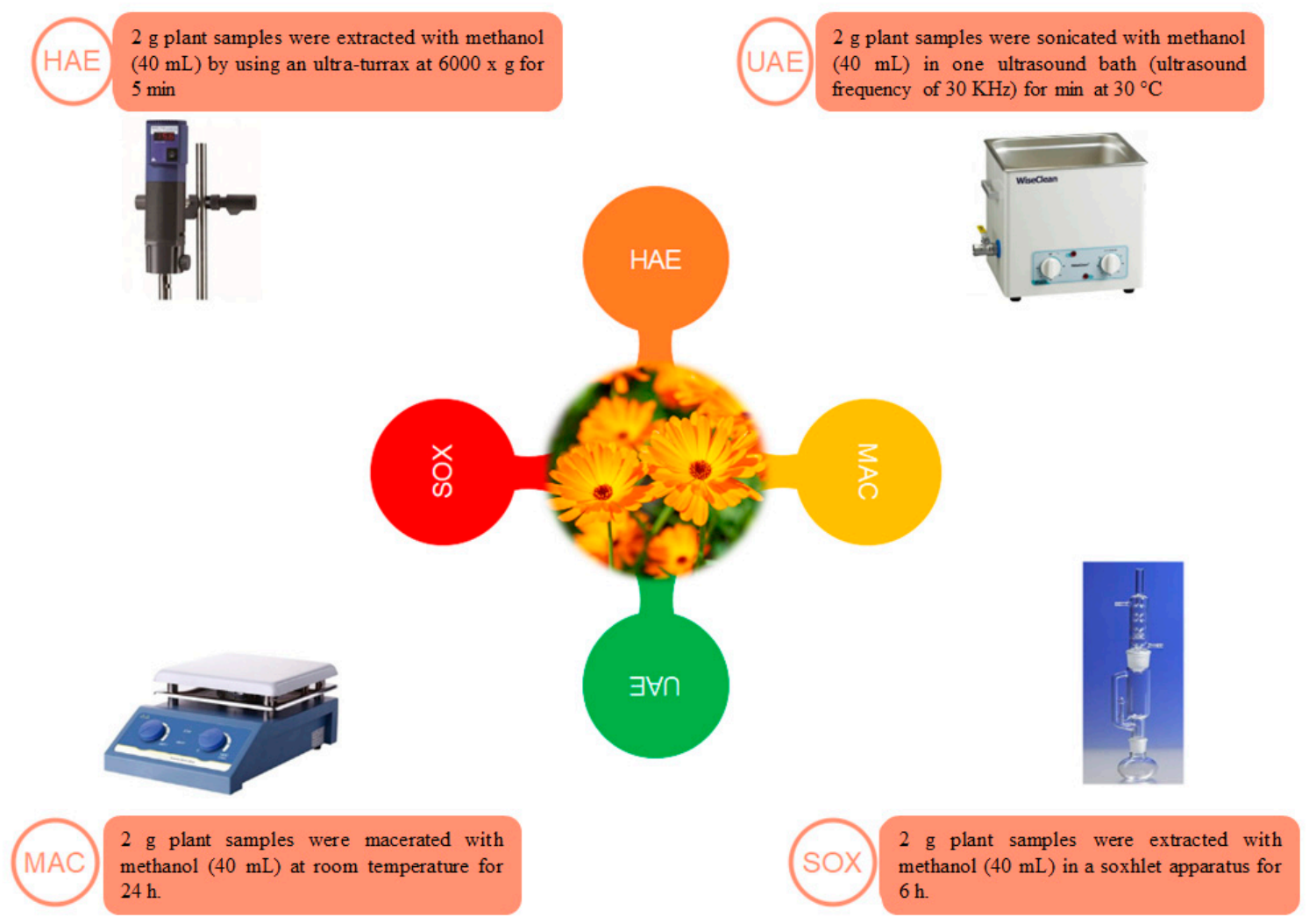

Figure 1. The performed extraction procedures in the current study. 


\subsection{Profile of Bioactive Compounds}

The total phenolic, and flavonoid contents of the extracts were measured as previously described in detailed [18,19]. Briefly, in the total phenolic assay, the plant extracts $(50 \mu \mathrm{L}, 1 \mathrm{mg} / \mathrm{mL})$ were mixed with Folin-Ciocalteu reagents (100 $\mu \mathrm{L}$ (diluted 1/9 with water) Merck) and then sodium carbonate added ( $75 \mu \mathrm{L}, 2 \%$ aqueous solution, Sigma-Aldrich, St. Louis, MO, USA). After $120 \mathrm{~min}$ incubation at room temperature, the absorbances were measured at $765 \mathrm{~nm}$. Regarding total flavonoid content assay, the plant extracts $(200 \mu \mathrm{L}, 1 \mathrm{mg} / \mathrm{mL})$ were mixed with aluminum chloride ( $2 \%$ methanolic solution, Sigma-Aldrich). After $15 \mathrm{~min}$ incubation at room temperature, the absorbances were read at $415 \mathrm{~nm}$. Results were expressed as equivalent of standards, namely gallic acid (GAE) for phenolics, and rutin (RE) for flavonoids.

\subsection{Mass Spectrometer and Chromatograph Conditions}

The instrument system used for the quantitative screening of 53 phytochemicals in the studied extracts was a Shimadzu brand liquid chromatography-mass spectrometry (LCMS)-8040 model tandem mass spectrometer coupled with a Nexera model ultra-high performance liquid chromatograph (U-HPLC, Shimadzu, Kyoto, Japan). Binary pumps (LC 30AD), a column oven (CTO 10 ASvp), an autosampler (SIL $30 \mathrm{AC}$ ) and a degasser (DGU $20 \mathrm{~A} 3 \mathrm{R}$ ) were the components of the separation system (the chromatograph). The analyses were performed by a previously developed and validated liquid chromatography-mass spectrometry/mass spectrometry LC-MS/MS method [20]. The detailed analytical parameters of the applied validated method were given in Supplementary Materials.

\subsection{Antioxidant and Enzyme Inhibitory Assays}

Reducing power, metal chelating, phosphomolybdenum and free radical scavenging assays were preferred as antioxidant assays. Standard compounds including trolox (TE/g extract) and ethylenediamine tetraacetate (EDTAE/g extract) were used to express antioxidant abilities. Enzyme inhibitory abilities were tested against different enzymes including $\alpha$-glucosidase, $\alpha$-amylase, cholinesterases and tyrosinase. Standard inhibitors namely acarbose, kojic acid and galantamine were used to express the enzyme inhibition abilities (mg standard equivalent/g extract). The details for these assays were given in our earlier paper [21].

\subsection{Data Analysis}

Data were reported as mean \pm standard deviation. Means comparison of total bioactive compounds and pharmaceutical activities were done using one-way anova. When significant occurred (at $p<0.05)$, post-hoc test were achieved using Tukey's test. Hierarchical cluster analysis was achieved to assess whether distinct clusters with homogeneous running patterns can be pinpoint among the studied samples. The Euclidean distance and Ward's linkage method were employed. Following the hierarchical cluster analysis (HCA) and partial least squares-discriminant analysis (PLS-DA) was performed to highlight the pharmaceutical activities mostly varied from a cluster to another. Finally, the pharmaceutical activities characteristic of each clusters were identified. The statistical evaluation was performed on $\mathrm{R}$ version 3.6.2 software.

\section{Results and Discussion}

\subsection{Chemical Profile}

Table 1 reports the amounts of phenolics and flavonoids in the tested extracts, as well as the results of phosphomolybenum assay. Total phenolic content was highest for the flower extracts, followed by the leaf extracts, and finally extracts from the roots. A previous investigation reported that the methanol extract of $C$. officinalis flower possessed highest phenolic and flavonoid contents as compared 
to the corresponding leaf extract [22]. Regarding the flavonoid content, the leaf extracts showed highest content, followed by extracts from the flower and roots (Table 1).

Table 1. Total phenolic (TPC), flavonoid (TFC) and antioxidant capacity of the tested extracts.

\begin{tabular}{|c|c|c|c|c|}
\hline Parts & Methods & TPC (mg GAE/g extract) & TFC (mg RE/g extract) & PBD ( mmol TE/g extract) \\
\hline \multirow{4}{*}{ Flowers } & HAE & $32.18 \pm 0.40^{b}$ & $28.48 \pm 0.40^{\mathrm{d}}$ & $2.10 \pm 0.08^{a}$ \\
\hline & MAC & $34.27 \pm 0.40^{\mathrm{a}}$ & $28.38 \pm 0.45^{\mathrm{d}}$ & $2.14 \pm 0.13^{\mathrm{a}}$ \\
\hline & UAE & $32.32 \pm 0.40^{b}$ & $28.41 \pm 0.50^{\mathrm{d}}$ & $2.08 \pm 0.02^{\mathrm{a}}$ \\
\hline & SOX & $30.96 \pm 0.17^{c}$ & $32.50 \pm 0.67^{b, c}$ & $1.68 \pm 0.02^{b, c, d}$ \\
\hline \multirow{4}{*}{ Leaves } & HAE & $23.57 \pm 0.30^{f}$ & $31.60 \pm 0.74^{\mathrm{c}}$ & $1.91 \pm 0.09 \mathrm{a}, \mathrm{b}$ \\
\hline & MAC & $25.20 \pm 0.26^{\mathrm{e}}$ & $28.33 \pm 0.14^{\mathrm{d}}$ & $1.66 \pm 0.07^{\mathrm{c}, \mathrm{d}}$ \\
\hline & UAE & $24.39 \pm 0.14^{\mathrm{e}, \mathrm{f}}$ & $32.95 \pm 0.35^{b}$ & $1.84 \pm 0.06^{b, c}$ \\
\hline & SOX & $27.93 \pm 0.25^{\mathrm{d}}$ & $34.61 \pm 0.29^{a}$ & $1.52 \pm 0.11^{\mathrm{d}, \mathrm{e}}$ \\
\hline \multirow{4}{*}{ Roots } & HAE & $18.40 \pm 0.37 \mathrm{~g}$ & $2.54 \pm 0.09^{\mathrm{e}}$ & $1.29 \pm 0.09^{\mathrm{e}, \mathrm{f}, \mathrm{g}}$ \\
\hline & MAC & $19.27 \pm 0.25^{g}$ & $2.45 \pm 0.12^{\mathrm{e}}$ & $1.26 \pm 0.05^{\mathrm{f}, \mathrm{g}}$ \\
\hline & UAE & $16.49 \pm 0.39^{h}$ & $2.45 \pm 0.07^{\mathrm{e}}$ & $1.49 \pm 0.09^{\mathrm{d}, \mathrm{e}, \mathrm{f}}$ \\
\hline & SOX & $17.36 \pm 0.19^{h}$ & $2.62 \pm 0.06^{\mathrm{e}}$ & $1.17 \pm 0.08^{g}$ \\
\hline
\end{tabular}

Values are reported as mean \pm S.D. PBD: Phosphomolybdneum assay; HAE: Homogenizer assisted extraction; MAC: Maceration; UAE: Ultrasound assisted extraction; SOX: Soxhlet. GAE: Gallic acid equivalent; RE: Rutin equivalent; TE: Trolox equivalent. Different superscripts $(a-h)$ in the same column indicate significantly differences among the tested extracts $(p<0.05$, by ANOVA assay).

Table 2 shows detailed chemical composition of the different extracts along with the concentration of specific compounds present. It was noted that extraction method influenced the biochemical composition of extracts. For instance, fumaric acid concentration was highest in flower extract obtained by Soxhlet extraction; catechin was identified in the flower and root extracts obtained by Soxhlet extraction only. Hesperidin, astragalin, and rutin were identified in the flower and leaves only. Hesperidin and rutin concentration were highest in the flower extracts (3.076-2.618 and 4.810-3.656 $\mathrm{mg} / \mathrm{g}$ extract, for hesperidin and rutin, respectively). Caffeic acid concentration was highest in Soxhlet extracts $(0.635,0.963$, and 0.734 , for the flower, leaf, and root, respectively), suggesting that Soxhlet extraction might be more suitable for caffeic acid extraction. Nicotiflorin was identified in the flower extracts only while amentoflavone occurred only in the roots (Table 2). Cosmosiin was extracted from C. officinalis leaves using conventional maceration and Soxhlet extraction methods. The flower extracts obtained by homogenizer assisted, maceration, and ultrasound assisted extraction showed highest total antioxidant capacity (2.14-2.08 $\mathrm{mmol} \mathrm{TE} / \mathrm{g}$ extract). In the literature, the different level of total and individual compounds in the different parts of $C$. officinalis were reported by several authors [6,23-26]. The different level could be explained by climatic, geographical and genetic factors. Taken together, C. officinalis might be regarded as a potential raw material for bioactive constituents. 
Table 2. Chemical composition of the tested extracts (mg/g extract).

\begin{tabular}{|c|c|c|c|c|c|c|c|c|c|c|c|c|}
\hline Analyte & 1 & 2 & 3 & 4 & 5 & 6 & 7 & 8 & 9 & 10 & 11 & 12 \\
\hline Vanillin & N.D. & N.D. & N.D. & N.D. & N.D. & N.D. & N.D. & N.D. & N.D. & N.D. & N.D. & N.D. \\
\hline Daidzin & N.D. & N.D. & N.D. & N.D. & N.D. & N.D. & N.D. & N.D. & N.D. & N.D. & N.D. & N.D. \\
\hline Piceid & N.D. & N.D. & N.D. & N.D. & N.D. & N.D. & N.D. & N.D. & N.D. & N.D. & N.D. & N.D. \\
\hline Coumarin & N.D. & N.D. & N.D. & N.D. & N.D. & N.D. & N.D. & N.D. & N.D. & N.D. & N.D. & N.D. \\
\hline Hesperidin & 2.964 & 3.076 & 3.091 & 2.618 & 0.343 & 0.264 & 0.284 & 0.297 & N.D. & N.D. & N.D. & N.D. \\
\hline Quinic acid & 8.757 & 6.362 & 6.322 & 13.426 & 4.847 & 4.889 & 4.262 & 6.804 & 4.329 & 4.646 & 4.072 & 5.896 \\
\hline Fumaric aid & 3.739 & 3.758 & 3.141 & 10.322 & 5.122 & 5.077 & 4.154 & 1.145 & 1.129 & 5.236 & 3.255 & 8.746 \\
\hline Aconitic acid & N.D. & N.D. & N.D. & N.D. & N.D. & N.D. & N.D. & N.D. & N.D. & N.D. & N.D. & N.D. \\
\hline Gallic acid & N.D. & N.D. & N.D. & N.D. & N.D. & N.D. & N.D. & N.D. & N.D. & N.D. & N.D. & N.D. \\
\hline Protocatechuic acid & 0.084 & 0.124 & 0.121 & 0.120 & 0.074 & 0.090 & 0.075 & 0.112 & 0.089 & 0.112 & 0.066 & 0.127 \\
\hline Gentisic acid & N.D. & N.D. & N.D. & N.D. & N.D. & N.D. & N.D. & N.D. & N.D. & N.D. & N.D. & N.D. \\
\hline Epigallocatechin & N.D. & N.D. & N.D. & N.D. & N.D. & N.D. & N.D. & N.D. & N.D. & N.D. & N.D. & N.D. \\
\hline Protocatechuic aldehyde & N.D. & N.D. & 0.028 & 0.023 & 0.043 & 0.048 & 0.046 & 0.061 & 0.072 & 0.076 & 0.045 & 0.083 \\
\hline Catechin & N.D. & N.D. & N.D. & 0.088 & N.D. & N.D. & N.D. & N.D. & N.D. & N.D. & N.D. & 0.025 \\
\hline Chlorogenic acid & 8.555 & 9.214 & 8.532 & 8.313 & 8.748 & 8.575 & 7.036 & 10.118 & 4.901 & 4.835 & 3.767 & 4.869 \\
\hline Tannic acid & N.D. & N.D. & N.D. & N.D. & N.D. & N.D. & N.D. & N.D. & N.D. & N.D. & N.D. & N.D. \\
\hline 4-OH Benzoic acid & N.D. & N.D. & N.D. & N.D. & N.D. & N.D. & N.D. & N.D. & N.D. & N.D. & N.D. & N.D. \\
\hline Epigallocatechin gallate & N.D. & N.D. & N.D. & N.D. & N.D. & N.D. & N.D. & N.D. & N.D. & N.D. & N.D. & N.D. \\
\hline Cynarin & N.D. & N.D. & N.D. & N.D. & N.D. & N.D. & N.D. & N.D. & N.D. & 0.042 & N.D. & N.D. \\
\hline Vanilic acid & N.D. & N.D. & N.D. & N.D. & N.D. & N.D. & N.D. & N.D. & N.D. & N.D. & N.D. & N.D. \\
\hline Epicatechin & N.D. & N.D. & N.D. & N.D. & N.D. & N.D. & N.D. & N.D. & N.D. & N.D. & N.D. & N.D. \\
\hline Caffeic acid & 0.405 & 0.535 & 0.477 & 0.635 & 0.571 & 0.610 & 0.472 & 0.963 & 0.422 & 0.572 & 0.357 & 0.734 \\
\hline Syringic acid & N.D. & N.D. & N.D. & N.D. & N.D. & N.D. & N.D. & N.D. & N.D. & N.D. & N.D. & N.D. \\
\hline Syringic aldehyde & N.D. & N.D. & N.D. & N.D. & N.D. & N.D. & N.D. & N.D. & N.D. & N.D. & N.D. & N.D. \\
\hline Epicatechin gallate & N.D. & N.D. & N.D. & N.D. & N.D. & N.D. & N.D. & N.D. & N.D. & N.D. & N.D. & N.D. \\
\hline$p$-Coumaric acid & N.D. & N.D. & N.D. & N.D. & N.D. & N.D. & N.D. & N.D. & N.D. & N.D. & N.D. & N.D. \\
\hline Sinapic acid & N.D. & N.D. & N.D. & N.D. & N.D. & N.D. & N.D. & N.D. & N.D. & N.D. & N.D. & N.D. \\
\hline Ferulic acid & 0.084 & N.D. & N.D. & N.D. & N.D. & N.D. & N.D. & N.D. & N.D. & N.D. & N.D. & N.D. \\
\hline Salicylic acid & 0.398 & 0.649 & 0.561 & 0.570 & 0.463 & 0.670 & 0.393 & 0.724 & 0.192 & 0.302 & 0.229 & 0.350 \\
\hline Cyranoside & N.D. & N.D. & N.D. & N.D. & N.D. & N.D. & N.D. & N.D. & N.D. & N.D. & N.D. & N.D. \\
\hline Miquelianin & 0.015 & N.D. & 0.015 & 0.023 & 0.003 & 0.013 & 0.011 & 0.005 & 0.004 & 0.006 & 0.007 & 0.005 \\
\hline isoquercitrin & 2.645 & 3.706 & 2.600 & 5.512 & 9.778 & 13.562 & 7.056 & 24.907 & 0.996 & 0.421 & 0.207 & 0.545 \\
\hline
\end{tabular}


Table 2. Cont.

\begin{tabular}{|c|c|c|c|c|c|c|c|c|c|c|c|c|}
\hline Analyte & 1 & 2 & 3 & 4 & 5 & 6 & 7 & 8 & 9 & 10 & 11 & 12 \\
\hline Rutin & 3.844 & 4.351 & 4.810 & 3.656 & 0.557 & 0.365 & 0.458 & 0.518 & N.D. & N.D. & N.D. & N.D. \\
\hline Genistin & N.D. & N.D. & N.D. & N.D. & N.D. & N.D. & N.D. & N.D. & N.D. & N.D. & N.D. & N.D. \\
\hline$o$-Coumaric acid & N.D. & N.D. & N.D. & N.D. & N.D. & N.D. & N.D. & N.D. & N.D. & N.D. & N.D. & N.D. \\
\hline Ellagic acid & N.D. & N.D. & N.D. & N.D. & N.D. & N.D. & N.D. & N.D. & N.D. & N.D. & N.D. & N.D. \\
\hline Rosmarinic acid & N.D. & N.D. & N.D. & N.D. & N.D. & N.D. & N.D. & N.D. & N.D. & N.D. & N.D. & N.D. \\
\hline Fisetin & N.D. & N.D. & N.D. & N.D. & N.D. & N.D. & N.D. & N.D. & N.D. & N.D. & N.D. & N.D. \\
\hline Cosmosiin & N.D. & N.D. & N.D. & N.D. & N.D. & 0.038 & N.D. & 0.051 & N.D. & N.D. & N.D. & N.D. \\
\hline Quercitrin & N.D. & N.D. & N.D. & N.D. & N.D. & N.D. & N.D. & N.D. & N.D. & N.D. & N.D. & N.D. \\
\hline Astragalin & 0.135 & 0.174 & 0.129 & 0.250 & 0.327 & 0.350 & 0.204 & 0.629 & N.D. & N.D. & N.D. & N.D. \\
\hline Nicotiflorin & 0.484 & 0.537 & 0.570 & 0.468 & N.D. & N.D. & N.D. & N.D. & N.D. & N.D. & N.D. & N.D. \\
\hline Daidzein & N.D. & N.D. & N.D. & N.D. & N.D. & N.D. & N.D. & N.D. & N.D. & N.D. & N.D. & N.D. \\
\hline Genistein & N.D. & N.D. & N.D. & N.D. & N.D. & N.D. & N.D. & N.D. & N.D. & N.D. & N.D. & N.D. \\
\hline Quercetin & N.D. & 0.047 & 0.046 & 0.068 & 0.047 & 0.044 & 0.048 & 0.082 & N.D. & N.D. & N.D. & N.D. \\
\hline Luteolin & 0.003 & 0.004 & 0.006 & 0.005 & 0.007 & 0.008 & 0.008 & 0.010 & 0.003 & 0.003 & 0.002 & 0.002 \\
\hline Hesperetin & N.D. & N.D. & N.D. & N.D. & N.D. & N.D. & N.D. & N.D. & N.D. & N.D. & N.D. & N.D. \\
\hline Naringenin & N.D. & N.D. & N.D. & N.D. & N.D. & N.D. & N.D. & N.D. & N.D. & N.D. & N.D. & N.D. \\
\hline Kaempferol & N.D. & N.D. & N.D. & N.D. & N.D. & N.D. & N.D. & N.D. & N.D. & N.D. & N.D. & N.D. \\
\hline Apigenin & N.D. & N.D. & N.D. & N.D. & N.D. & N.D. & N.D. & N.D. & N.D. & N.D. & N.D. & N.D. \\
\hline Amentoflavone & N.D. & N.D. & N.D. & N.D. & N.D. & N.D. & N.D. & N.D. & 0.002 & 0.002 & 0.001 & 0.001 \\
\hline Acacetin & N.D. & N.D. & N.D. & N.D. & N.D. & N.D. & N.D. & N.D. & N.D. & N.D. & N.D. & N.D. \\
\hline Chrysin & N.D. & N.D. & N.D. & N.D. & N.D. & N.D. & N.D. & N.D. & N.D. & N.D. & N.D. & N.D. \\
\hline
\end{tabular}

N.D: Not determined. 1-Flowers-HAE; 2-Flowers-MAC; 3-Flowers-UAE; 4-Flowers-SOX; 5-Leaves-HAE; 6-Leaves-MAC; 7-Leaves-UAE; 8-Leaves-SOX; 9-Roots-HAE; 10-Roots-MAC; 11-Roots-UAE; 12-Roots-SOX. 


\subsection{Antioxidant Effects}

Several investigations have reported the antioxidant potential of $C$. officinalis. As an example, the ethanol extract of $C$. officinalis flower showed to reduce $\mathrm{Fe}^{3+}$ to $\mathrm{Fe}^{2+}$ (ferric reducing antioxidant power or FRAP assay) [27]. Ethanol extract of C. officinalis flower scavenged 2,2'-azino-bis(3-ethylbenzothiazoline-6-sulphonic acid) (ABTS) and 2,2-diphenyl-1-picrylhydrazyl $(\mathrm{DPPH})$ radicals at an $\mathrm{IC}_{50}$ of 6.5 and $100 \mu \mathrm{g} / \mathrm{mL}$, for respective assays [28]. In the present study, the flower extracts showed highest radical scavenging and reducing potential (Table 3). In general, the Soxhlet extract of $C$. officinalis flower $(37.58,57.69$, and $63.43 \mathrm{mg}$ TE/g, for DPPH, ABTS, and FRAP assays, respectively) showed highest equivalent values, meaning highest activity. The radical scavenging properties of natural compounds are often related to their ability to quench free radicals. As such, rutin and nicotiflorin, and isoquercitrin, identified in higher concentration in C. officinalis flower have been shown to scavenge free radicals [29]. Extracts of $C$. officinalis roots were the least active. C. officinalis leaf extracts showed highest chelating activity. Relevantly, isoquercitrin, identified in high level in C. officinalis leaf Soxhlet extract ( $24.907 \mathrm{mg} / \mathrm{g}$ extract), has been reported to show remarkable chelating potential [30]. The pivotal role of oxidants in the onset, progression, and exacerbation of human diseases has rendered antioxidant determination crucial for the development of novel drug candidates. In fact, the ability of a molecule to exhibit antioxidant potential as well as interacting with other biological pathways is an attractive therapeutic strategy. We observed some results for antioxidant properties of $C$. officinalis. For example, Petkova et al [25] reported antioxidant properties of infusion and decoction from flowers of C. officinalis via different chemical methods such as DPPH, ABTS, FRAP, and cupric reducing power (CUPRAC) and the authors noted that the extracts exhibited moderate efficiency. Also, Ourabia et al [24] reported moderate activity of C. officinalis on ABTS and DPPH assays. Pires et al [31] studied the antioxidant activities of hydroalcoholic and infusion of C. officinalis flowers with DPPH and FRAP assays and the hydroalcoholic one exhibited stronger ability when compared infusion. Marian et al [32] also reported antioxidant properties of hydroalcoholic extract from $C$. officinalis flowers. These findings could provide that the alcohol could be useful for the preparing novel products from $C$. officinalis, to increase efficiency.

Table 3. Antioxidant properties of the tested extracts.

\begin{tabular}{|c|c|c|c|c|c|c|}
\hline Parts & Methods & $\begin{array}{l}\text { DPPH (mg } \\
\text { TE/g extract) }\end{array}$ & $\begin{array}{l}\text { ABTS (mg } \\
\text { TE/g extract) }\end{array}$ & $\begin{array}{l}\text { CUPRAC (mg } \\
\text { TE/g extract) }\end{array}$ & $\begin{array}{l}\text { FRAP (mg } \\
\text { TE/g extract) }\end{array}$ & $\begin{array}{l}\text { Metal Chelating (mg } \\
\text { EDTAE/g extract) }\end{array}$ \\
\hline \multirow{3}{*}{ Flowers } & HAE & $35.90 \pm 0.06^{b, c}$ & $46.11 \pm 0.59^{b}$ & $107.53 \pm 2.97^{\mathrm{a}, \mathrm{b}}$ & $55.94 \pm 0.41^{\mathrm{c}}$ & $12.58 \pm 0.24 \mathrm{~d}, \mathrm{e}$ \\
\hline & UAE & $32.18 \pm 0.31^{\mathrm{d}}$ & $46.61 \pm 0.27^{b}$ & $103.11 \pm 1.29^{b}$ & $52.75 \pm 1.21^{\mathrm{d}}$ & $10.79 \pm 0.17^{\mathrm{e}}$ \\
\hline & SOX & $37.58 \pm 0.69^{a}$ & $57.69 \pm 0.64^{a}$ & $106.21 \pm 0.19^{a, b}$ & $63.43 \pm 1.41^{\mathrm{a}}$ & $16.95 \pm 0.47^{\mathrm{c}}$ \\
\hline \multirow{2}{*}{ Leaves } & UAE & $28.07 \pm 0.19^{f}$ & $35.37 \pm 0.73^{d}$ & $83.69 \pm 1.18^{d}$ & $44.91 \pm 0.71^{\mathrm{d}}$ & $21.65 \pm 0.87^{b}$ \\
\hline & SOX & $36.78 \pm 0.21^{\mathrm{a}, \mathrm{b}}$ & $47.16 \pm 0.81^{b}$ & $89.74 \pm 1.63^{c}$ & $51.63 \pm 0.36^{\mathrm{d}, e}$ & $26.11 \pm 0.40^{\mathrm{a}}$ \\
\hline \multirow{2}{*}{ Roots } & HAE & $24.02 \pm 0.37 g$ & $30.95 \pm 1.02^{\mathrm{e}}$ & $57.65 \pm 0.37^{f}$ & $35.37 \pm 0.04^{h}$ & $13.02 \pm 0.32 \mathrm{~d}, \mathrm{e}$ \\
\hline & MAC & $20.57 \pm 0.25^{h}$ & $32.60 \pm 0.07^{\mathrm{e}}$ & $57.91 \pm 0.75^{\mathrm{f}}$ & $35.37 \pm 0.97^{h}$ & $13.04 \pm 1.14^{\mathrm{d}, \mathrm{e}}$ \\
\hline
\end{tabular}

Values are reported as mean \pm S.D. HAE: Homogenizer assisted extraction; MAC: Maceration; UAE: Ultrasound assisted extraction; SOX: Soxhlet. TE: Trolox equivalent: EDTAE: EDTA equivalent. Different superscripts (a-i) in the same column indicate significantly differences among the tested extracts $(p<0.05$, by ANOVA assay).

\subsection{Enzyme Inhibitory Properties}

Current pharmacological approach to treat Alzheimer's disease includes cholinesterase inhibitors administration in order to maintain normal level of acetylcholine [33]. Both acetyl (AChE) and butyryl $(\mathrm{BChE})$ cholinesterase catalyze the hydrolysis of acetylcholine thereby terminating cholinergic signals in the nervous system. In the current investigation, the root extract of $C$. officinalis obtained by maceration 
showed highest inhibition against AChE (2.54 mg GALAE/g extract) while the homogenizer assisted extract showed highest inhibition against BChE (6.08 mg GALAE/g extract). Ercetin et al [22] reported that the different solvent extracts (n-hexane, dicholoromethane, acetone, ethyl acetate, methanol and water) from C. officinalis leaves and flowers exhibited the low inhibition values on AChE and BChE. The ethyl acetate extracts exhibited the best inhibitory effects among them and the findings could indicate that the plant has a complex phytochemical profile. Amentoflavone, identified in the roots, was previously identified in other plants possessing cholinesterase inhibitory properties [34,35]. Amentoflavone inhibited AChE in scopolamine-induced memory impaired mice [36]. Cynarin, characterized from the maceration extract of $C$. officinalis roots $(0.042 \mathrm{mg} / \mathrm{g}$ extract), was previously reported to fit to $\mathrm{AChE}$ catalytic triad [37]. The hydroxycinnamic acid derivative was reported to inhibit AChE with an $\mathrm{IC}_{50}$ value of $243.67 \mathrm{nM}$ [38]. The ability of the $C$. officinalis extracts to inhibit tyrosinase was also investigated. Topical cream enriched with $C$. officinalis ethanol extract was reported to reduce skin melanin content [39]. However, the authors did not mention the exact plant part used for this study. Ethanolic extract of the leaves of $C$. officinalis was reported to possess $\alpha$-amylase inhibitory activity [40]. However, findings gathered from this study showed that $C$. officinalis extracts exhibited weak inhibition against $\alpha$-amylase and $\alpha$-glucosidase. C. officinalis flower extract exhibited $<30 \%$ and $<45 \%$ inhibition at $20 \mathrm{mg} / \mathrm{mL}$ and $500 \mu \mathrm{g} / \mathrm{mL}$, for $\alpha$-amylase and $\alpha$-glucosidase, respectively [40]. As shown in Table 4 , in general the flower extract showed highest inhibition against $\alpha$-amylase, followed by the leaf extracts and finally the root extracts, while the root extracts were more active against $\alpha$-glucosidase and flower extracts were the least active. Following our literature survey, it was noted that scientific information regarding the enzyme inhibition properties of $C$. officinalis is scarce. Thus, our presented findings could provide valuable information for novel applications with C. officinalis.

Table 4. Enzyme inhibitory properties of the tested extracts.

\begin{tabular}{|c|c|c|c|c|c|c|}
\hline Parts & Methods & $\begin{array}{c}\text { AChE } \\
\text { Inhibition } \\
\text { (mg GALAE/g } \\
\text { extract) }\end{array}$ & $\begin{array}{c}\text { BChE } \\
\text { Inhibition } \\
\text { (mg GALAE/g } \\
\text { extract) }\end{array}$ & $\begin{array}{c}\text { Tyrosinase } \\
\text { Inhibition (mg } \\
\text { KAE/g extract) }\end{array}$ & $\begin{array}{c}\text { Amylase } \\
\text { Inhibition } \\
\text { (mmol ACAE/g } \\
\text { extract) }\end{array}$ & $\begin{array}{c}\text { Glucosidase } \\
\text { Inhibition } \\
\text { (mmol ACAE/g } \\
\text { extract) }\end{array}$ \\
\hline \multirow{4}{*}{ Flowers } & HAE & $1.52 \pm 0.11^{\mathrm{d}}$ & $2.83 \pm 0.36^{\mathrm{d}, \mathrm{e}, \mathrm{f}}$ & $69.86 \pm 0.46^{c}$ & $0.25 \pm 0.01^{a}$ & $0.05 \pm 0.02^{f}$ \\
\hline & MAC & $2.05 \pm 0.29^{b, c}$ & $2.20 \pm 0.45^{\mathrm{f}}$ & $70.16 \pm 0.41^{\mathrm{c}}$ & $0.24 \pm 0.01^{\mathrm{a}, \mathrm{b}}$ & $0.09 \pm 0.01^{\mathrm{f}}$ \\
\hline & UAE & $1.94 \pm 0.13^{c}$ & $2.42 \pm 0.12^{f}$ & $70.97 \pm 0.36^{c}$ & $0.24 \pm 0.01^{\mathrm{a}, \mathrm{b}}$ & $0.51 \pm 0.06^{\mathrm{e}}$ \\
\hline & SOX & $2.08 \pm 0.19^{b, c}$ & $0.76 \pm 0.09 g$ & $69.83 \pm 0.82^{c}$ & $0.23 \pm 0.01^{b}$ & $0.66 \pm 0.02^{c, d}$ \\
\hline \multirow{4}{*}{ Leaves } & HAE & na & $3.48 \pm 0.28^{\mathrm{c}, \mathrm{d}}$ & $72.82 \pm 0.27^{b}$ & $0.20 \pm 0.01^{\mathrm{c}}$ & $0.75 \pm 0.01^{b, c}$ \\
\hline & MAC & na & $4.37 \pm 0.20^{b}$ & $73.95 \pm 0.33^{a, b}$ & $0.18 \pm 0.01^{\mathrm{d}, \mathrm{e}}$ & $0.75 \pm 0.01 \mathrm{~b}, \mathrm{c}, \mathrm{d}$ \\
\hline & UAE & $2.16^{b, c}$ & $3.84 \pm 0.20^{b, c}$ & $72.84 \pm 0.03^{b}$ & $0.20 \pm 0.01^{c, d}$ & $0.75 \pm 0.01^{\mathrm{a}, \mathrm{b}, \mathrm{c}}$ \\
\hline & SOX & na & $2.81 \pm 0.06^{\mathrm{d}, \mathrm{e}, \mathrm{f}}$ & $69.66 \pm 0.76^{c}$ & $0.21 \pm 0.01^{\mathrm{c}}$ & $0.65 \pm 0.09^{d}$ \\
\hline \multirow{4}{*}{ Roots } & HAE & na & $6.08 \pm 0.28^{a}$ & $74.05 \pm 0.41^{\mathrm{a}, \mathrm{b}}$ & $0.18 \pm 0.01^{\mathrm{d}, \mathrm{e}}$ & $0.79 \pm 0.01^{\mathrm{a}, \mathrm{b}}$ \\
\hline & MAC & $2.54 \pm 0.08^{a}$ & $3.43 \pm 0.21^{c, d, e}$ & $74.43 \pm 1.31^{\mathrm{a}, \mathrm{b}}$ & $0.17 \pm 0.01^{\mathrm{e}}$ & $0.85 \pm 0.01^{\mathrm{a}}$ \\
\hline & UAE & na & $5.72 \pm 0.62^{\mathrm{a}}$ & $74.66 \pm 0.08^{a}$ & $0.18 \pm 0.01^{\mathrm{e}}$ & $0.83 \pm 0.01^{a, b}$ \\
\hline & SOX & $2.38 \pm 0.14^{\mathrm{a}, \mathrm{b}}$ & $2.57 \pm 0.08^{\mathrm{e}, \mathrm{f}}$ & $70.50 \pm 0.29^{c}$ & $0.18 \pm 0.01 \mathrm{~d}, \mathrm{e}$ & $0.83 \pm 0.01^{\mathrm{a}, \mathrm{b}}$ \\
\hline
\end{tabular}

Values are reported as mean \pm S.D. HAE: Homogenizer assisted extraction; MAC: Maceration; UAE: Ultrasound assisted extraction; SOX: Soxhlet. GALAE: Galatamine equivalent; KAE: Kojic acid equivalent; ACAE: Acarbose equivalent. na: Not active. Different superscripts $(\mathrm{a}-\mathrm{g})$ in the same column indicate significantly differences among the tested extracts $(p<0.05$, by ANOVA assay).

\subsection{Multivariate Analysis}

All samples were clustered using hierarchical clustered analysis in consideration of their antioxidant properties and enzyme inhibitory abilities. The purpose of this analysis was to figure out groups of samples that are relatively homogeneous and therefore that share specific biological activities in common. At the outset, the optimal number of cluster in the data set was determined by the estimation of the variance within the cluster. As shown in Figure 2A, a bend was observed at $\mathrm{k}$ equal to 3 which meant that added clusters beyond the third have little value. Thus, the suggested number of clusters was 3 . Figure 2B showed the Clusters depiction of HCA; as can be seen the samples 
was consolidated depending on the plant parts. Cluster 1 comprised of the samples derived from the flower part, cluster 2 included the samples of the leaves part while cluster 3 was an assemblage of roots samples. The clustering structure was acceptable since the average silhouette width was close to 1 .

HCA approach shown that $C$. officinalis biological activities were mainly influenced by the organ's nature; however, no information related to how the biological activities mostly varied from a part to another were identified. Therefore, applying partial least squares discriminant analysis, can help to provide the information on these aspects. Figure 3 depicted the outcomes of PLS-DA by considering the type of parts as class memberships. According to Figure 2A, a complete separation from the three plant parts was observed along the first two function of the model. The discrimination result was the same with HCA, with an excellent predicted value (Area Under Curve (AUC)) for the first two function was 1). The biological activities mostly influenced by the type of part were identified based on the variable's importance in projection. Eight biological assays i.e., metal chelating, anti-amylase anti-glucosidase and all the antioxidant assays had a variable importance in projection (VIP) score above 1; this suggested that the results of that assays varied considerably from an organ to another.

The present study revealed that the distribution of phytoconstituents as well as the derived biological activities of $C$. officinalis differ significantly between the different parts. This observation is in line with that of [41] who indicated that the synthesis and accumulation of the molecules varied to one organs to another of the same plant, as a consequence of their differential gene expression which by extension impacts the pharmaceutical activities of the plant extracts. Much further according to these authors reported that the amount and composition of bioactive compounds differ at the sub-cellular level as well as within plant tissues. On the other hand, del Baño, et al. [42] mentioned that the differential distribution of phenolic compounds in the different part of plants is due to their endogeneous biosynthesis and within their transport to one organ to another as well. When compared the biological activities of the different organs, the flower extracts showed the higher total antioxidant content and free radical scavenging (ABTS and DPPH), power reducing (FRAP and CUPRAC) and anti-amylase activities compared to the extracts of the other organs (Figure 4). Nevertheless, overall weak BChE, tyrosinase, metal chelating activity (MCA) and glucosidase activities were detected for flowers extracts. Higher metal chelating activity were obtained with the leaves extracts whereas their anti-tyrosinase and anti-glucosidase activities were close to those of the roots' extracts. Despite our findings that the biological activities of C. officinalis were predominantly influenced by the type of organs, it is worth noting that within organs there is a remarkable extraction process effect on the biological activities. Numerous publications reported the variation of the nature and yield of biocompounds as well as the bioactivities of the extracts obtained using different extraction techniques [43-46]. It is well known that biologically active molecules occur in low concentration in plant materials. Thus, extraction is a crucial in the discovery of biologically-active molecules from herbal materials. 


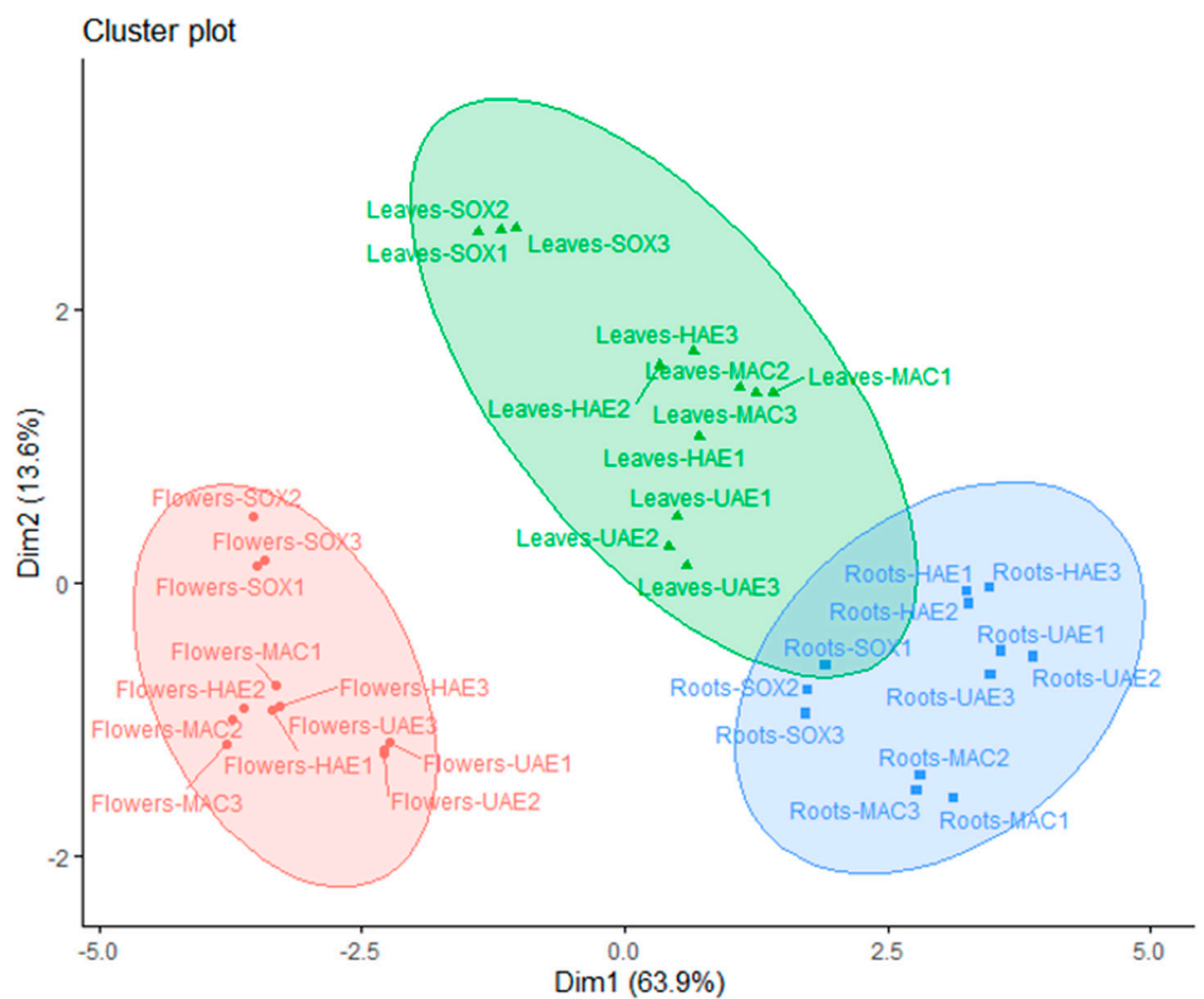

B

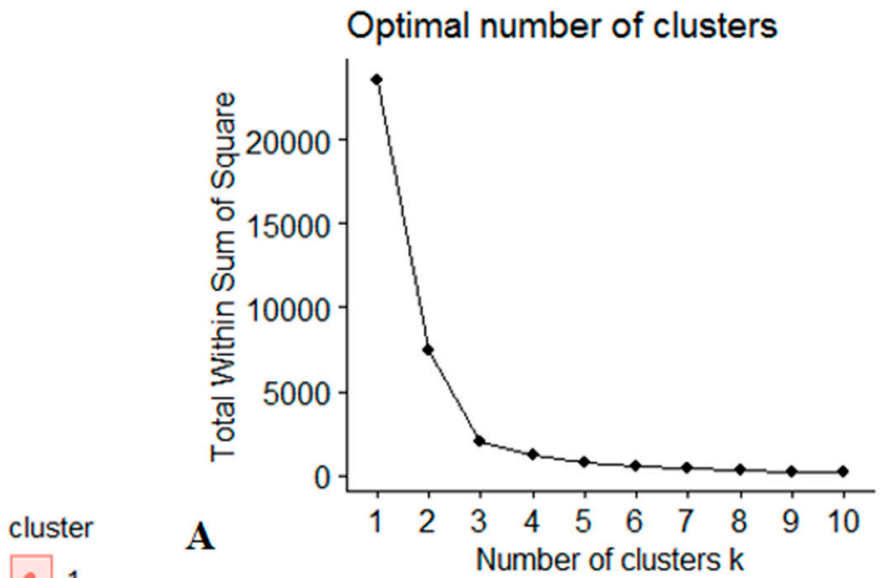

Clusters silhouette plot

Average silhouette width: 0.68

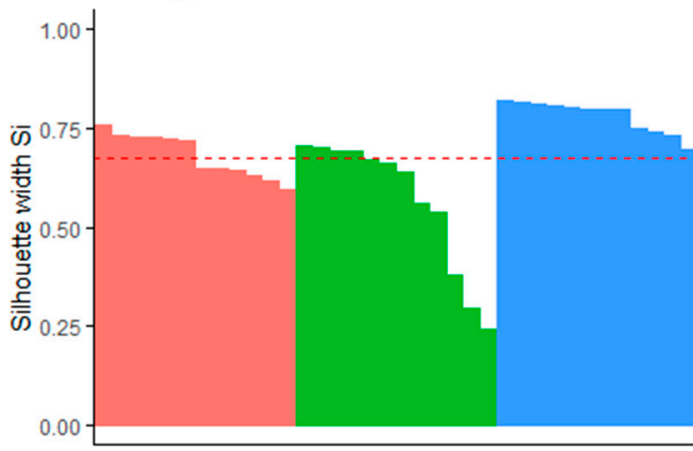

C

Figure 2. Hierarchical cluster analysis on the biological activities of Calendula officinalis samples. (A): Estimation of the optimal number of clusters. (B): Cluster plot. (C): Assessment of the goodness of the cluster analysis using the average distance between the clusters. 

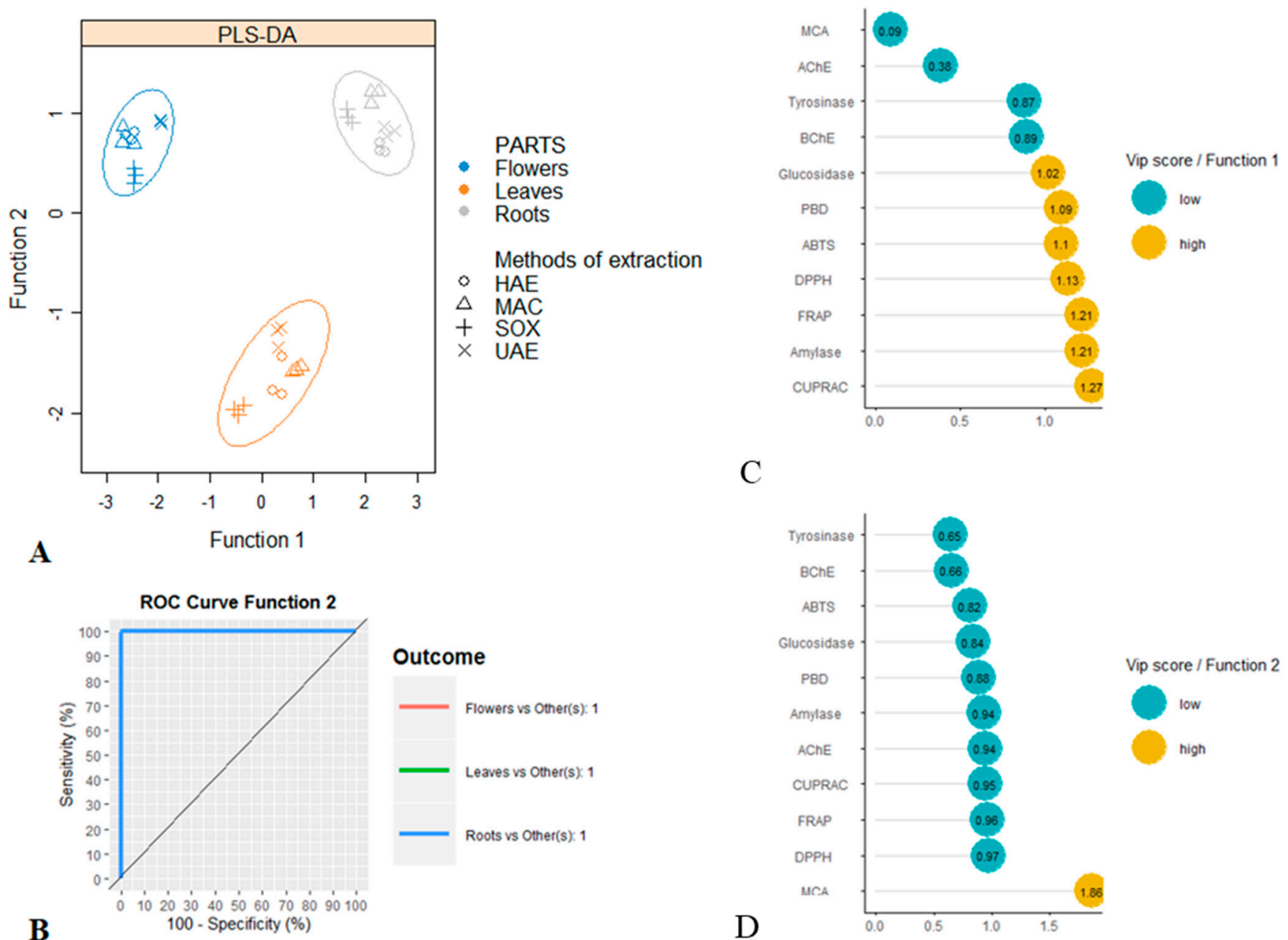

Figure 3. (A) Samples plot of supervised partial least squares discriminant analysis. (B) Area under the curve average and receiver operating characteristic curve using one-vs-all comparisons reflecting the robustness of the supervised model. (C,D) Discriminant biological activities evaluation by VIP approach for the first two function of the discriminant model. 

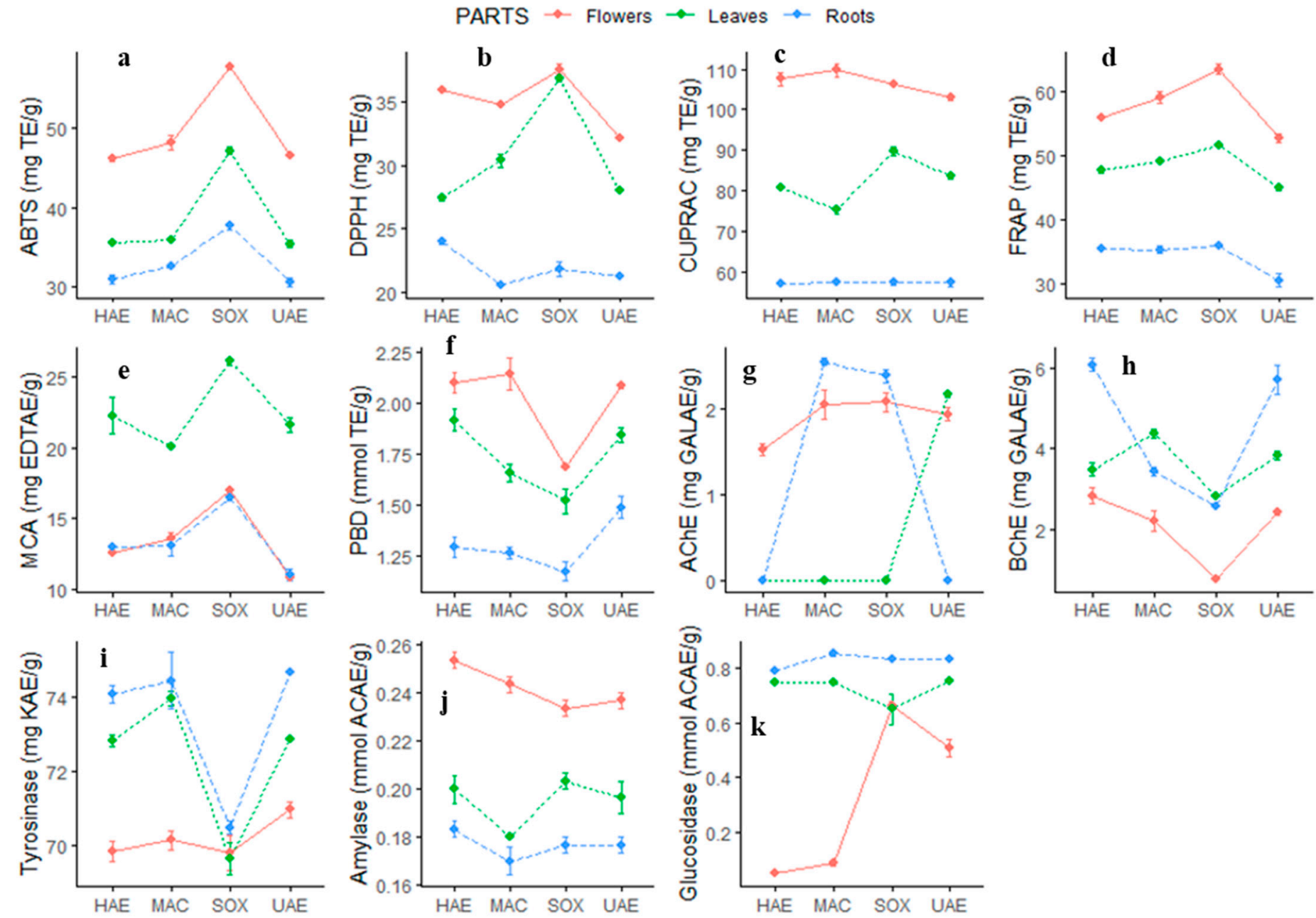

Figure 4. Comparison of antioxidant properties and enzyme inhibitory capacities of the different organs of C. officinalis. (a) ABTS radical scavenging, (b) DPPH radical scavenging, (c) CUPRAC assay, (d) FRAP assay, (e) Metal chelating (MCA) assay, (f) Phosphomolybdenum (PBD) assay, (g) AChE inhibition, (h) BChE inhibition, (i) Tyrosinase inhibition, (j) Amylase inhibition, (k) Glucosidase inhibition. 


\section{Conclusions}

Data presented here clearly showed that extraction method influenced the phytochemical composition of herbal extracts and that phytochemical profile varied from one plant part to the other. In the current study, the flower extract of $C$. officinalis demonstrated the best scavenging effect and reducing potential. However, the leaf extract exhibited the highest metal chelating potential. Extracts obtained by Soxhlet extraction were associated with potent antioxidant propensities. The root extracts of $C$. officinalis exhibited potent inhibitory potential against cholinesterases and tyrosinase.

Supplementary Materials: The following are available online at http://www.mdpi.com/2076-3417/10/17/5920/s1, Table S1. Analytical method validation parameters that belong to the LC-MS/MS method.

Author Contributions: Conceptualization, G.A., G.Z., K.I.S.; Methodology, G.Z., O.C., S.B., M.A.Y.; software, G.A., K.I.S; validation, G.Z., M.C.N.P.-A., M.F.M; formal analysis, G.Z; investigation, G.A., G.Z., M.G., D.M.; resources, G.A., G.Z.; data curation, G.Z., M.C.N.P.-A., M.F.M.; writing-original draft preparation, G.Z.; M.C.N.P.-A., M.F.M; writing-review and editing, M.G., D.M.; visualization, K.I.S; supervision, G.Z.; project administration, G.Z; funding acquisition, M.G., D.M. All authors have read and agreed to the published version of the manuscript.

Funding: This research received no external funding

Conflicts of Interest: The authors declare no conflict of interest.

\section{References}

1. Vieira da Silva, B.; Barreira, J.C.M.; Oliveira, M.B.P.P. Natural phytochemicals and probiotics as bioactive ingredients for functional foods: Extraction, biochemistry and protected-delivery technologies. Trends Food Sci. Technol. 2016, 50, 144-158. [CrossRef]

2. Belwal, T.; Ezzat, S.M.; Rastrelli, L.; Bhatt, I.D.; Daglia, M.; Baldi, A.; Devkota, H.P.; Orhan, I.E.; Patra, J.K.; Das, G. A critical analysis of extraction techniques used for botanicals: Trends, priorities, industrial uses and optimization strategies. TrAC Trends Anal. Chem. 2018, 100, 82-102. [CrossRef]

3. Mishra, A.K.; Mishra, A.; Pragya; Chattopadhyay, P. Screening of acute and sub-chronic dermal toxicity of Calendula officinalis L. essential oil. Regul. Toxicol. Pharmacol. 2018, 98, 184-189. [CrossRef]

4. Efstratiou, E.; Hussain, A.I.; Nigam, P.S.; Moore, J.E.; Ayub, M.A.; Rao, J.R. Antimicrobial activity of Calendula officinalis petal extracts against fungi, as well as Gram-negative and Gram-positive clinical pathogens. Complement. Ther. Clin. Pract. 2012, 18, 173-176. [CrossRef]

5. Cruceriu, D.; Diaconeasa, Z.; Socaci, S.; Socaciu, C.; Rakosy-Tican, E.; Balacescu, O. Biochemical profile, selective cytotoxicity and molecular effects of Calendula officinalis extracts on breast cancer cell lines. Not. Bot. Horti Agrobot. Cluj-Napoca 2020, 48, 24-39. [CrossRef]

6. Escher, G.B.; Borges, L.D.C.; Santos, J.S.; Cruz, T.M.; Marques, M.B.; do Carmo, M.A.V.; Azevedo, L.; Furtado, M.M.; Sant'Ana, A.S.; Wen, M.C.; et al. From the field to the pot: Phytochemical and functional analyses of Calendula officinalis L. flower for incorporation in an organic yogurt. Antioxidants 2019, 8, 20.

7. Chitrakar, B.; Zhang, M.; Bhandari, B. Edible flowers with the common name "marigold": Their therapeutic values and processing. Trends Food Sci. Technol. 2019, 89, 76-87. [CrossRef]

8. Hamburger, M.; Adler, S.; Baumann, D.; Förg, A.; Weinreich, B. Preparative purification of the major anti-inflammatory triterpenoid esters from Marigold (Calendula officinalis). Fitoterapia 2003, 74, 328-338. [CrossRef]

9. Neukirch, H.; D'Ambrosio, M.; Via, J.D.; Guerriero, A. Simultaneous quantitative determination of eight triterpenoid monoesters from flowers of 10 varieties of Calendula officinalis L. and characterisation of a new triterpenoid monoester. Phytochem. Anal. 2004, 15, 30-35. [CrossRef]

10. Ohmiya, A. Diversity of carotenoid composition in flower petals. Jarq-Jpn. Ag. Res. Q. 2011, 45, $163-171$. [CrossRef]

11. Petrović, L.; Lepojevic, Z.; Sovilj, V.; Adamović, D.; Tešević, V. An investigation of $\mathrm{CO}_{2}$ extraction of marigold (Calendula officinalis L.). J. Serb. Chem. Soc. 2007, 72, 407-413. [CrossRef]

12. Kozlowska, J.; Stachowiak, N.; Prus, W. Stability studies of collagen-based microspheres with Calendula officinalis flower extract. Polym. Degradation Stab. 2019, 163, 214-219. [CrossRef]

13. Cioinac, S.E. Use of calendula cream balm to medicate the feet of diabetic patients: Case series. Int. J. Nurs. Stud. 2016, 3, 102-112. [CrossRef] 
14. Pedram Rad, Z.; Mokhtari, J.; Abbasi, M. Calendula officinalis extract/PCL/Zein/Gum arabic nanofibrous bio-composite scaffolds via suspension, two-nozzle and multilayer electrospinning for skin tissue engineering. Int. J. Biol. Macromol. 2019, 135, 530-543. [CrossRef]

15. Slavov, A.; Ognyanov, M.; Vasileva, I. Pectic polysaccharides extracted from pot marigold (Calendula officinalis) industrial waste. Food Hydrocoll. 2020, 101, 105545. [CrossRef]

16. Pires, T.C.S.P.; Dias, M.I.; Barros, L.; Ferreira, I.C.F.R. Nutritional and chemical characterization of edible petals and corresponding infusions: Valorization as new food ingredients. Food Chem. 2017, 220, 337-343. [CrossRef]

17. Hamzawy, M.A.; El-Denshary, E.S.M.; Hassan, N.S.; Mannaa, F.A.; Abdel-Wahhab, M.A. Dietary Supplementation of Calendula officinalis Counteracts the Oxidative Stress and Liver Damage Resulted from Aflatoxin. ISRN Nutr. 2013, 2013, 538427. [CrossRef]

18. Vladimir-Knežević, S.; Blažeković, B.; Bival Štefan, M.; Alegro, A.; Kőszegi, T.; Petrik, J. Antioxidant activities and polyphenolic contents of three selected Micromeria species from Croatia. Molecules 2011, 16, 1454-1470. [CrossRef]

19. Zengin, G.; Aktumsek, A. Investigation of antioxidant potentials of solvent extracts from different anatomical parts of Asphodeline anatolica E. Tuzlaci: An endemic plant to Turkey. Afr. J. Tradit. Complement. Altern. Med. 2014, 11, 481-488. [CrossRef]

20. Yilmaz, M.A. Simultaneous quantitative screening of 53 phytochemicals in 33 species of medicinal and aromatic plants: A detailed, robust and comprehensive LC-MS/MS method validation. Ind. Crop. Prod. 2020, 149, 112347. [CrossRef]

21. Grochowski, D.M.; Uysal, S.; Aktumsek, A.; Granica, S.; Zengin, G.; Ceylan, R.; Locatelli, M.; Tomczyk, M. In vitro enzyme inhibitory properties, antioxidant activities, and phytochemical profile of Potentilla thuringiaca. Phytochem. Lett. 2017, 20,365-372. [CrossRef]

22. Ercetin, T.; Senol, F.S.; Erdogan Orhan, I.; Toker, G. Comparative assessment of antioxidant and cholinesterase inhibitory properties of the marigold extracts from Calendula arvensis L. and Calendula officinalis L. Ind. Crop. Prod. 2012, 36, 203-208. [CrossRef]

23. BEKDEŞER, B. Modeling and optimizing microwave-assisted extraction of antioxidant compounds from marigold Calendula officinalis L. using response surface methodology. Turk. J. Chem. 2019, 43, 1457-1471. [CrossRef]

24. Ourabia, I.; Djebbar, R.; Tata, S.; Sabaou, N.; Fouial-Djebbar, D. Determination of essential oil composition, phenolic content, and antioxidant, antibacterial and antifungal activities of marigold (Calendula officinalis L.) cultivated in algeria. Carpath. J. Food Sci. Technol. 2019, 11, 93-110.

25. Petkova, D.; Mihaylova, D.; Denev, P.; Krastanov, A. Antioxidant activity of some edible flowers water extracts from Bulgaria. Bull. UASVM Food Sci. Technol. 2020, 77, 1. [CrossRef]

26. Sytar, O.; Hemmerich, I.; Zivcak, M.; Rauh, C.; Brestic, M. Comparative analysis of bioactive phenolic compounds composition from 26 medicinal plants. Saudi J. Biol. Sci. 2018, 25, 631-641. [CrossRef]

27. Babaee, N.; Moslemi, D.; Khalilpour, M.; Vejdani, F.; Moghadamnia, Y.; Bijani, A.; Baradaran, M.; Kazemi, M.T.; Khalilpour, A.; Pouramir, M.; et al. Antioxidant capacity of calendula officinalis flowers extract and prevention of radiation induced oropharyngeal mucositis in patients with head and neck cancers: A randomized controlled clinical study. Daru 2013, 21, 18. [CrossRef]

28. Preethi, K.C.; Kuttan, G.; Kuttan, R. Antioxidant potential of an extract of Calendula officinalis. flowers in vitro and in vivo. Pharm. Biol. 2006, 44, 691-697. [CrossRef]

29. Soberón, J.R.; Sgariglia, M.A.; Sampietro, D.A.; Quiroga, E.N.; Vattuone, M.A. Free radical scavenging activities and inhibition of inflammatory enzymes of phenolics isolated from Tripodanthus acutifolius. J. Ethnopharmacol. 2010, 130, 329-333. [CrossRef]

30. Catapano, M.C.; Tvrdý, V.; Karlíčková, J.; Migkos, T.; Valentová, K.; Křen, V.; Mladěnka, P. The stoichiometry of isoquercitrin complex with iron or copper is highly dependent on experimental conditions. Nutrients 2017, 9, 1193. [CrossRef]

31. Pires, T.C.S.P.; Dias, M.I.; Barros, L.; Calhelha, R.C.; Alves, M.J.; Oliveira, M.B.P.P.; Santos-Buelga, C.; Ferreira, I.C.F.R. Edible flowers as sources of phenolic compounds with bioactive potential. Food Res. Int. 2018, 105, 580-588. [CrossRef] [PubMed] 
32. Marian, E.; Vicaș, L.G.; Jurca, T.; Mureasan, M.; Stan, R.L.; Sevastre, B.; Diaconeasa, Z.; Ionescu, C.; Hangan, A.C. A comparative study on the biologic activity of Centaurea cyanus versus Calendula officinalis. Farmacia 2017, 65, 940-946.

33. Caratelli, V.; Ciampaglia, A.; Guiducci, J.; Sancesario, G.; Moscone, D.; Arduini, F. Precision medicine in Alzheimer's disease: An origami paper-based electrochemical device for cholinesterase inhibitors. Biosens. Bioelectron. 2020, 28, 112411. [CrossRef] [PubMed]

34. Erdogan-Orhan, I.; Altun, M.L.; Sever-Yilmaz, B.; Saltan, G. Anti-acetylcholinesterase and antioxidant assets of the major components (salicin, amentoflavone, and chlorogenic acid) and the extracts of Viburnum opulus and Viburnum lantana and their total phenol and flavonoid contents. J. Med. Food 2011, 14, 434-440. [CrossRef] [PubMed]

35. Eroglu Ozkan, E.; Yilmaz Ozden, T.; Ozsoy, N.; Mat, A. Evaluation of chemical composition, antioxidant and anti-acetylcholinesterase activities of Hypericum neurocalycinum and Hypericum malatyanum. S. Afr. J. Bot. 2018, 114, 104-110. [CrossRef]

36. Ishola, I.O.; Tota, S.; Adeyemi, O.O.; Agbaje, E.O.; Narender, T.; Shukla, R. Protective effect of Cnestis ferruginea and its active constituent on scopolamine-induced memory impairment in mice: A behavioral and biochemical study. Pharm. Biol. 2013, 51, 825-835. [CrossRef] [PubMed]

37. Silva, L.; Rodrigues, A.M.; Ciriani, M.; Falé, P.L.V.; Teixeira, V.; Madeira, P.; Machuqueiro, M.; Pacheco, R.; Florêncio, M.H.; Ascensão, L.; et al. Antiacetylcholinesterase activity and docking studies with chlorogenic acid, cynarin and arzanol from Helichrysum stoechas (Lamiaceae). Med. Chem. Res. 2017, 26, 2942-2950. [CrossRef]

38. Topal, M.; Gocer, H.; Topal, F.; Kalin, P.; Köse, L.P.; Gülçin, İ.; Çakmak, K.C.; Küçük, M.; Durmaz, L.; Gören, A.C.; et al. Antioxidant, antiradical, and anticholinergic properties of cynarin purified from the Illyrian thistle (Onopordum illyricum L.). J. Enzyme Inhib. Med. Chem. 2016, 31, 266-275. [CrossRef]

39. Akhtar, N.; Khan, B.A.; Haji, M.; Khan, S.; Ahmad, M.; Rasool, F.; Mahmood, T.; Rasul, A. Evaluation of various functional skin parameters using a topical cream of Calendula officinalis extract. Afr. J. Pharm. Pharmaco. 2011, 5, 199-206. [CrossRef]

40. Olennikov, D.N.; Kashchenko, N.I. Componential profile and amylase inhibiting activity of phenolic compounds from Calendula officinalis L. leaves. Sci. World J. 2014, 2014, 654193. [CrossRef]

41. Iloki-Assanga, S.B.; Lewis-Luján, L.M.; Lara-Espinoza, C.L.; Gil-Salido, A.A.; Fernandez-Angulo, D.; Rubio-Pino, J.L.; Haines, D.D. Solvent effects on phytochemical constituent profiles and antioxidant activities, using four different extraction formulations for analysis of Bucida buceras L. and Phoradendron californicum. BMC Res. Notes 2015, 8, 396. [CrossRef] [PubMed]

42. Del Baño, M.J.; Lorente, J.; Castillo, J.; Benavente-García, O.; del Río, J.A.; Ortuño, A.; Quirin, K.W.; Gerard, D. Phenolic diterpenes, flavones, and rosmarinic acid distribution during the development of leaves, flowers, stems, and roots of Rosmarinus officinalis. Antioxidant activity. J. Agric. Food Chem. 2003, 51, 4247-4253. [CrossRef] [PubMed]

43. Rajan, M.; Rajkumar, G.; Farias Lima Guedes, T.J.; Chagas Barros, R.G.; Narain, N. Performance of different solvents on extraction of bioactive compounds, antioxidant and cytotoxic activities in Phoenix loureiroi Kunth leaves. J. Appl. Res. Med. Aromat. Plants 2020, 17, 100247. [CrossRef]

44. Zhu, J.-J.; Yang, J.-J.; Wu, G.-J.; Jiang, J.-G. Comparative antioxidant, anticancer and antimicrobial activities of essential oils from Semen Platycladi by different extraction methods. Ind. Crop. Prod. 2020, 146, 112206. [CrossRef]

45. Daud, M.N.H.; Fatanah, D.N.; Abdullah, N.; Ahmad, R. Evaluation of antioxidant potential of Artocarpus heterophyllus L. J33 variety fruit waste from different extraction methods and identification of phenolic constituents by LCMS. Food Chem. 2017, 232, 621-632. [CrossRef]

46. Dall'Acqua, S.; Kumar, G.; Sinan, K.I.; Sut, S.; Ferrarese, I.; Mahomoodally, M.F.; Seebaluck-Sandoram, R.; Etienne, O.K.; Zengin, G. An insight into Cochlospermum planchonii extracts obtained by traditional and green extraction methods: Relation between chemical compositions and biological properties by multivariate analysis. Ind. Crop. Prod. 2020, 147, 112226. [CrossRef]

(C) 2020 by the authors. Licensee MDPI, Basel, Switzerland. This article is an open access article distributed under the terms and conditions of the Creative Commons Attribution (CC BY) license (http://creativecommons.org/licenses/by/4.0/). 